\title{
On-chip Direct Laser Writing of PAN-based Carbon Supercapacitor Electrodes
}

\author{
Andreas Hoffmann, Pablo Jiménez-Calvo, Volker Strauss* and Alexander J. C. Kuehne*
}

\author{
A. Hoffmann, Prof. Dr. A.J.C. Kuehne \\ Institute of Organic and Macromolecular Chemistry, Ulm University, Albert-Einstein-Allee 11, \\ 89081 Ulm, Germany. \\ E-mail: alexander.kuehne@uni-ulm.de
}

Dr. Pablo Jiménez-Calvo, Dr. V. Strauss

Department of Colloid Chemistry, Max-Planck-Institute of Colloids and Interfaces, Am

Mühlenberg 1, 14476 Potsdam, Germany.

E-mail: volker.strauss@mpikg.mpg.de

Keywords: polyacrylonitrile, carbonization, microcapacitor, laser-pyrolysis, laser-induced carbonization

\begin{abstract}
We report carbonization of polyacrylonitrile by direct laser writing to produce microsupercapacitors directly on-chip. We demonstrate the process by producing interdigitated carbon finger electrodes directly on a printed circuit board, which we then employ to characterize our supercapacitor electrodes. By varying the laser power, we are able to tune the process from carbonization to material ablation. This allows to not only convert pristine polyacrylonitrile films into carbon electrodes, but also to pattern and cut away non-carbonized material to produce completely freestanding carbon electrodes. While the carbon electrodes adhere well to the printed circuit board, non-carbonized polyacrylonitrile is peeled off the substrate. We achieve specific capacities as high as $260 \mu \mathrm{F} / \mathrm{cm}^{2}$ in a supercapacitor with 16 fingers.
\end{abstract}

\section{Introduction}

Miniaturized electric double layer capacitors - termed microsupercapacitors - enable integrated energy storage directly on chip, facilitating self-sufficient devices without large periphery for external power supply and management systems. ${ }^{1-3}$ Such devices for on-chip energy storage could potentially transform the way we employ wearable and textile electronics and foster the development of autonomous microrobots. ${ }^{4-6}$ Different classes of organic and inorganic 
materials have been explored for carbon-based microsupercapacitors electrodes, with the aim of increasing the surface area to yield acceptable capacitance despite their small size. ${ }^{7-11}$ However, fabrication techniques to produce the required electrode geometries are limited to off-chip methods. The solvents or high processing temperatures employed to obtain carbonized, conductive electrode material are incompatible with typical electronic chip materials. Of course, patterning and carbonization of carbon precursor materials remains possible off-chip; however, off-chip carbonization in turn requires precise and complicated placing, interfacing, and integration of the manufactured supercapacitor on the dedicated chip. ${ }^{12}$

We have previously reported on laser-induced carbonization of polyacrylonitrile (PAN) carbonfibers and other carbon-precursors. ${ }^{13-16}$. Conventional carbonization of PAN is performed in convection ovens at temperatures $>1000{ }^{\circ} \mathrm{C} .{ }^{17,18}$ By contrast, laser-induced carbonization is beneficial, as heat is locally produced in the focus of the laser, preventing exposure to high temperatures in undesired areas. Moreover, laser-carbonization is fast, potentially allowing pattern formation of carbonized PAN when scanning the laser focus across a film. ${ }^{19}$

However, direct laser-carbonization of PAN has not been employed on PAN films until now. Realization of this technology would allow for miniaturized and precisely structured carbon electrodes directly on-chip, without the need for adhesives or precise positioning and interfacing.

Here, we demonstrate how interdigitated carbon electrodes can be directly laser-patterned into a film of PAN to produce microsupercapacitors. We perform direct laser-writing on commercially available printed circuit boards (PCB) to demonstrate that the laser-written carbon structures are well interfaced with the metallic current collectors on the PCB and that our laser process does not interfere with the materials of the substrate. The resulting supercapacitor electrodes are then characterized and benchmarked using an ionic liquid electrolyte.

\section{Results and Discussion}

PAN is a transparent polymer, which shows no absorption in the visible and near infrared spectrum. To enable laser carbonization, an absorber is added, to facilitate absorption of the laser energy and transfer heat into the exposed PAN material. We have previously employed graphene nanoplatelets as absorbers ${ }^{13}$ however, these fillers strongly increase the viscosity of the PAN solution, which is beneficial for fiber spinning but not necessarily beneficial for casting films. Therefore, we here make use of carbon nanotubes (CNTs), which come as a dispersion in DMSO. CNTs exhibit high conductivity, which is why we do not expect 
detrimental effects of the filler on the conductivity of the carbonized PAN material. We employ a concentration of $3 \mathrm{wt} \%$ of CNT per weight PAN, while the concentration of PAN in DMSO is $12 \mathrm{wt} \%$. The concentration of $3 \mathrm{wt} \% \mathrm{CNT}$ is optimized for sufficient absorption at the laser wavelength $\left(\mathrm{CO}_{2}, 10.6 \mu \mathrm{m}\right)$ (see Figure $\mathrm{S} 1$ in the Supporting Information). We can spin-coat this PAN/CNT solution in DMSO onto almost any desired flat substrate and the resulting film thickness can be precisely tuned through the speed of rotation or the concentration of PAN in solution (see Figure S2 in the Supporting Information). However, to avoid coating of the entire chip or PCB board with PAN/CNT in undesired areas, we here turn to pipette printing and subsequent doctor blading of the PAN/CNT solution. This way we are able to precisely deposit PAN/CNT onto the desired areas. As a substrate, we employ PCB with square silver collector electrodes of $16 \mathrm{~mm}^{2}$ in area, which are separated by a distance of $7.5 \mathrm{~mm}$. The collector electrodes are connected to a potentiostat via banana clips, which fit drillings in the PCB (see Figure 1).

a
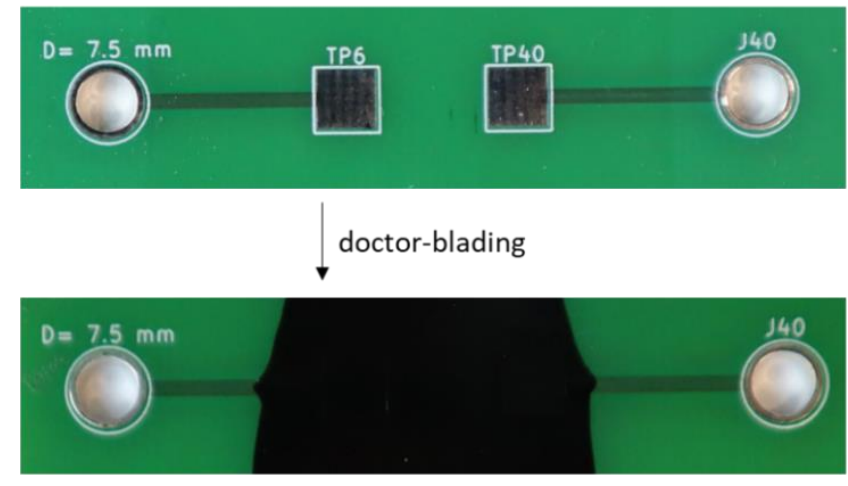

laser-carbonization

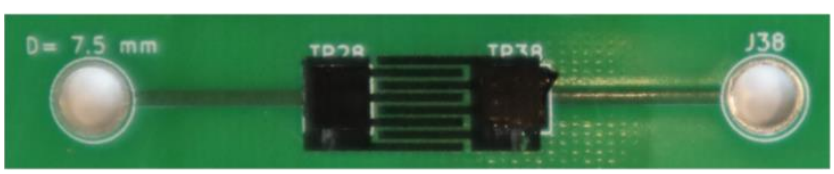

b

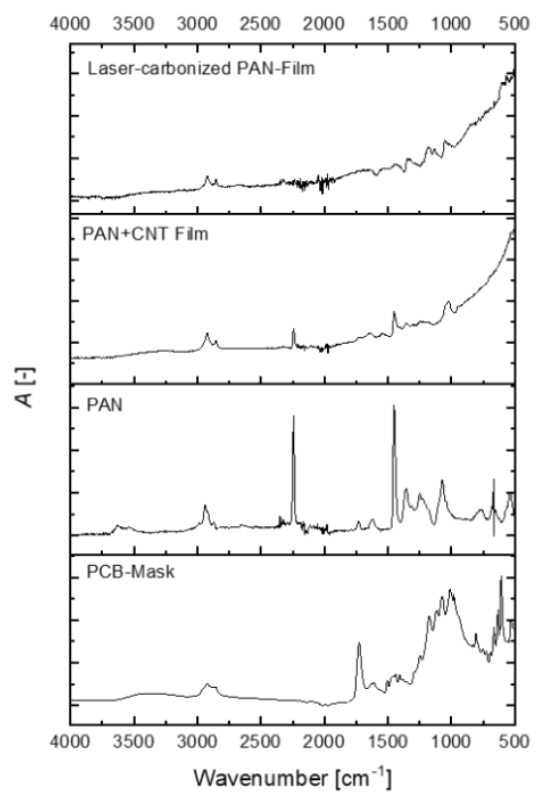

Figure 1. a) Photographs of the microcaps during various manufacturing stages. Bare PCBs containing a square silver electrode, which acts as a current collector. The current-collector connects the carbon electrodes to a read-out device via banana clips. The space in between, as well as the current collectors themselves, are coated with a PAN-CNT film. In a second step, the film is laser-carbonized to interdigitated electrodes. b) FT-IR-absorbance apectra of the PCB-mask, the PAN, the laser-absorbing PAN-CNT composite film and the laser-carbonized electrodes. 
We deposit the viscous PAN/CNT solution onto the PCB, by pipetting $2.5 \mathrm{~mL}$ of the viscous PAN/CNT solution as a grid of individual droplets. Closed films are drawn by doctor blading with a $30 \mu \mathrm{m}$ gap into an approximately $3 \mathrm{~cm}$ long stripe across the collector electrodes. The resulting films have a thickness of 20 - $21 \mu \mathrm{m}$ after drying (see Figure 1a).

These films are irradiated with a $\mathrm{CO}_{2}$ laser with a wavelength of $10.6 \mu \mathrm{m}$. In an attempt to replicate the thermal two-step treatment of stabilization and carbonization, we conduct laser exposure at increasing power density in air to generate laser-induced stabilization. However, we are not able to separate the exothermic stabilization event from carbonization. In contrast to carbonization, where increasing laser power density produces higher degrees of carbonization, for the stabilization step there seems to exist a sharp threshold of $0.26 \mathrm{~J} / \mathrm{mm}^{2}$. Below this threshold we do not see a change in the material properties or the infrared spectrum, in which stabilization is detectable by the disappearance of the corresponding nitrile band at $2442 \mathrm{~cm}^{-1}$ (see Figure 1b). Above the threshold we obtain conductive structures, indicating that we can conduct stabilization and carbonization in one step, despite the presence of air. Typically, thermal carbonization is conducted in the absence of air to avoid oxidation of the carbon material to $\mathrm{CO}_{2}$. When we further increase the laser dose to $0.7 \mathrm{~J} / \mathrm{mm}^{2}$ then we do no longer observe converted carbon structures, but instead we observe the bare substrate, indicating the occurrence of an ablation process rather than pyrolytic carbonization (see Figure 2). This interval in power density is beneficial, as we can perform precise carbonization within this parameter window, while we can also employ the ablation process with power densities beyond this interval for precise removal of the PAN-precursor films. The underlying PCB board is not harmed by either process, as no visible or measurable material changes in infrared spectroscopy or conductivity occur.

We carry on to pattern interdigitated electrodes with eight or 16 fingers at $0.63 \mathrm{~J} / \mathrm{mm}^{2}$, which is ideal for carbonization. In a second patterning step, we increase the power density to ablation and follow a trace between the electrode fingers to remove any non-carbonized material between the carbonized electrode fingers (see Figure 3). 


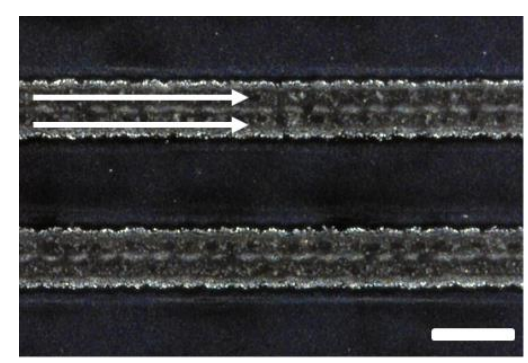

0.63

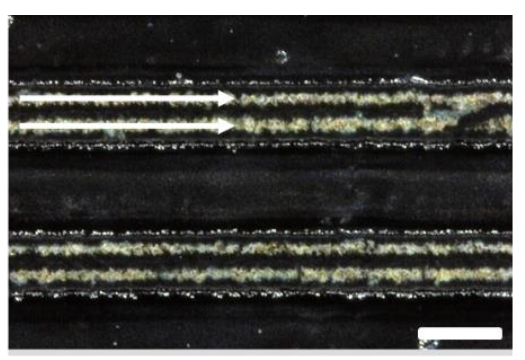

0.70

dose $\left[\mathrm{J} / \mathrm{mm}^{2}\right]$

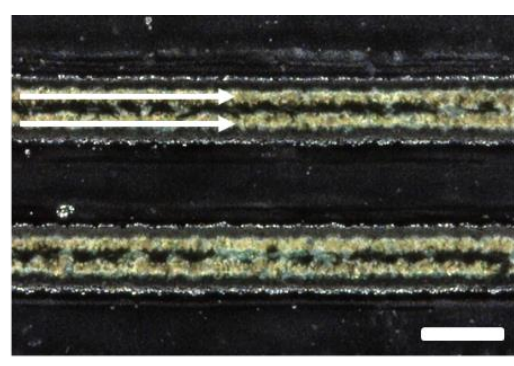

1.17

Figure 2. Variation of the laser dose for carbonization or ablation of PAN-films. White arrows indicate the pathway of the laser beam. A laser dose of $0.63 \mathrm{~J} / \mathrm{mm}^{2}$ lead to carbonized structures, whereas increasing the dose to $0.70 \mathrm{~J} / \mathrm{mm}^{2}$ leads to ablation of PAN material revealing the yellow/golden color of the PCB board. Doses higher than $1.17 \mathrm{~J} / \mathrm{mm}^{2}$ lead large area ablation, which limits the quality of the remaining PAN and carbon patterns. The scale bars represent $200 \mu \mathrm{m}$.

Now that the carbonized electrode pattern is separated from the pristine area, the excessive noncarbonized PAN film can simply be peeled off the PCB (see Figure 3a). By contrast, the carbonized interdigitating supercapacitor electrodes adhere to the PCB (see Figure $3 \mathrm{a}$ and Video in the Supporting Information). This way, the entire electrode structures including the sides of the electrode fingers down to the PCB substrate become accessible for the electrolyte in the final supercapacitor device. For 16 finger electrodes, the individual fingers are produced by scanning twice with the laser beam, followed by two additional scans for ablation and separation of the carbonized electrodes from the pristine PAN film (see Figure 3b-d). The eight finger devices are produced by seven laser traces next to each other (see Figure 3e). For eight fingers, the electrode width is $w=510 \mu \mathrm{m}$ with a height of $h=9.4 \mu \mathrm{m}$ at a spacing of $d=290 \mu \mathrm{m}$ between electrodes; and for 16 fingers the dimensions are $w=145 \mu \mathrm{m}, h=10.9 \mu \mathrm{m}$ and $d=263 \mu \mathrm{m}$ (see Figure S3). The laser carbonization process produces electrodes of hierarchical porosity (see Figure 4a,b). This is a well-known feature of the fast laser carbonization process, where the gasses produced during carbonization are formed instantaneously, leading to the porous foam-like structure, with high surface area, which is beneficial for the capacitor performance. $^{13}$ 
a
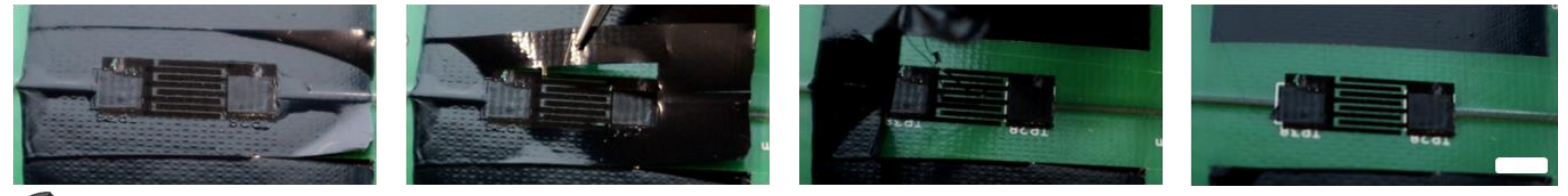

$r$

b
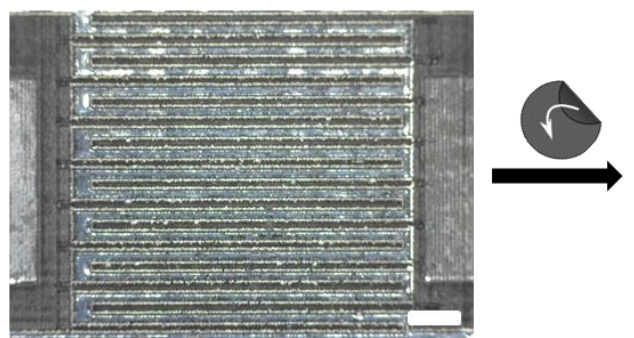

c

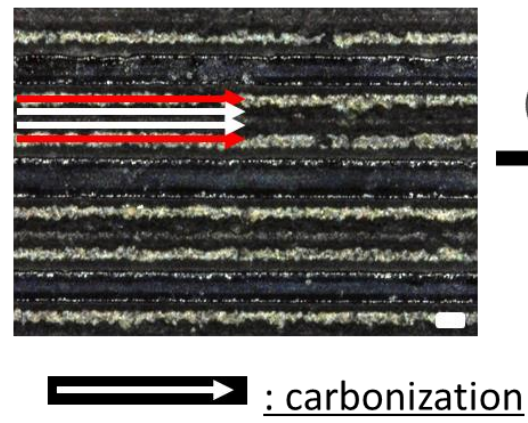

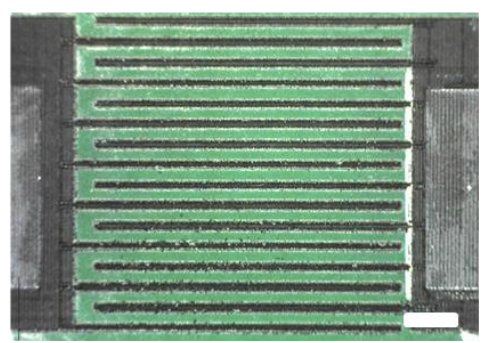

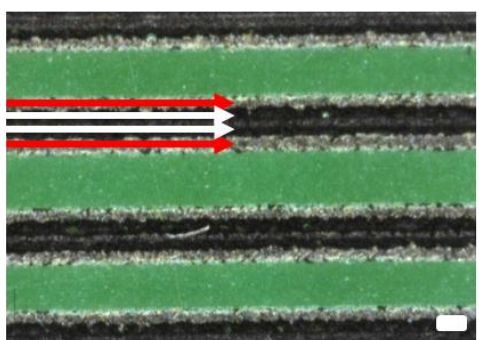

: ablation

Figure 3. Laser carbonization and ablation to separate carbonized pattern from non-carbonized film: a) The non-carbonized film is removed by peeling off with a pair of tweezers. The carbonized interdigitated electrode structure remains attached on the PCB. The scale bar represents $5 \mathrm{~mm}$. b) Micrographs of the laser carbonized and ablated films before and after removal of the non-carbonized film. The scale bar represent $1 \mathrm{~mm}$. c) Close-up on 4 electrodes and arrows indicating the laser paths for carbonization (white arrow) and ablation (red arrow) before and after removal of the non-carbonized PAN film. Scale bars indicate $100 \mu \mathrm{m}$. d) Scanning electron images of microcap electrodes of 16-finger microcaps and e) 8-finger microcaps. The white arrows indicate the laser pathways for carbonization, producing electrodes of different widths. The scale bar indicates $100 \mu \mathrm{m}$.

Next, we test the performance of electrodes in electrochemical measurements. As electrolyte we employ $20 \mu \mathrm{L}$ of the ionic liquid 1-ethyl-3-methylimidazolium bis(trifluoromethylsulfonyl) imide (EMIM TFSI), which we deposit on the interdigitated finger electrodes in an inert nitrogen atmosphere. After an equilibration period of $12 \mathrm{~h}$, we conduct electrochemical impedance spectroscopy and cyclovoltammetry. Nyquist plots of our eight or 16 finger 
electrodes show a semicircle at high frequencies and a steep increase with $85^{\circ}$ phase angle at lower frequencies, indicating capacitive behavior (see Figure 4c). The semicircles intercept the $\mathrm{x}$-axis at $2.3 \mathrm{k} \Omega$ for the eight finger electrodes and at $2.8 \mathrm{k} \Omega$ for the 16 finger electrodes. We interpret these values as the electrode resistance $R_{\mathrm{e}}$ (see Figure $4 \mathrm{c}$ ). ${ }^{20}$ By contrast, the charge transfer resistance $R_{\mathrm{CT}}$ is increased for the 16-finger microsupercapacitor (170 k $\Omega$ ) compared to the eight-finger device $(116 \mathrm{k} \Omega)$, which we account to the increased surface area in the 16 finger device (see Figure 4c). For the same reason, cyclovoltammetry show that the double layer capacitance $C_{\mathrm{sp}}$ of the 16 -finger device is much higher than in the 8-fingered device (see Figure 4d).
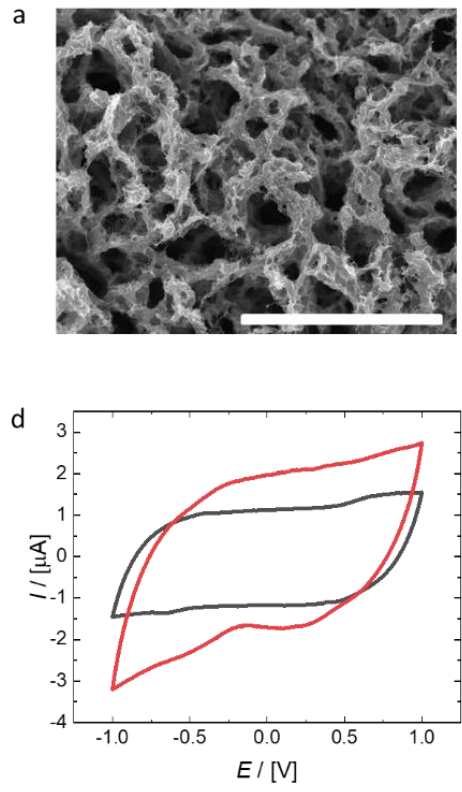

b
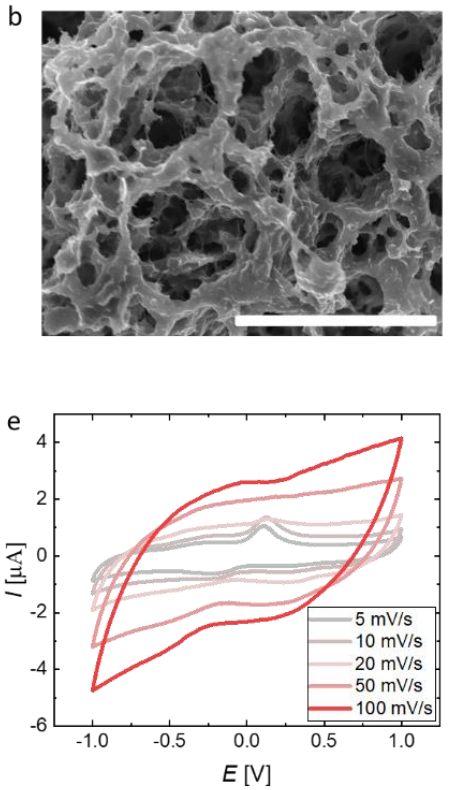
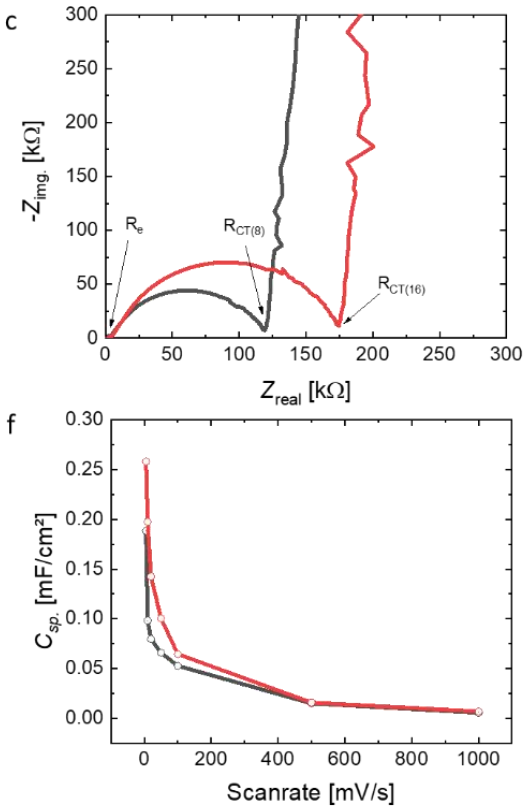

Figure 4. a) SEM-images of laser carbonized electrodes of the 8-finger and b) 16-finger pattern. The scale bars indicate $5 \mu \mathrm{m}$. c) Nyquist plots of laser-carbonized microcaps with 8- (black) and 16-finger (red) pattern. d) Cyclic voltammograms of 8- (black) and 16- finger (red) microcaps, recorded at $50 \mathrm{mV} / \mathrm{s}$. e) Cyclic voltammograms of 16-finger microcaps, recorded at various scan rates. f) Specific capacitance of 8- (black) and 16-finger (red) microcaps at scan rates from 5 to $1000 \mathrm{mV} / \mathrm{s}$.

When we plot the specific area capacitance $C_{\mathrm{sp}}$ against the scan rate, we observe a similar trend for the 8 and 16-fingered electrode devices. However, the microsupercapacitor with 16-fingers achieves significantly higher $C_{\mathrm{sp}}=260 \mu \mathrm{F} / \mathrm{cm}^{2}$ at low scan rates compared to $C_{\mathrm{sp}}=190 \mu \mathrm{F} / \mathrm{cm}^{2}$ in the 8-finger device (see Figure 4d,e and f). This difference in performance indicates that there might be a greater number of accessible pores in the 16 -finger device, which improves the 
capacitance compared to the 8-fingered microsupercapacitors with less overall interface. The achieved capacitances are of the same order of magnitude as for a recently reported graphene based finger capacitors of similar dimensions. ${ }^{7}$ Prolonged exposure of the electrodes to the ionic liquid electrolyte over the course of seven days, does not lead to their delamination or changes in their electrochemical performance.

\section{Conclusion}

We have demonstrated how to achieve direct on-chip laser-carbonization of PAN films. Our specifically designed process was used to fabricate carbon supercapacitor electrodes. We can precisely tune the process from carbonization to ablation, allowing us to remove any noncarbonized material to obtain freestanding interdigitating electrodes.

The developed process gives access to highly integrated energy storage devices of on-chip applications, which in the future might become even further miniaturized. Tuning of the exact porosity and potential post carbonization treatment to further activate the materials to increase the porosity might lead to even larger capacitances and better performance.

\section{Experimental Section/Methods}

Materials: Acrylonitrile homopolymer $\left(\mathrm{M}_{\mathrm{w}}=150.000 \mathrm{~g} \mathrm{~mol}^{-1}\right)$ was obtained from Dralon GmbH, Dimethysulfoxide (DMSO) was obtained from Fisher Scientific, Acetone was obtained from Sigma-Aldrich, a CNT dispersion in DMSO with a weight content of $2 \mathrm{wt} \%$ CNTs was obtained from Future Carbon $\mathrm{GmbH}$ and 1-Ethyl-3-methylimidazolium bis(trifluoromethylsulfonyl)imide (EMIM TFSI; 99.5\%) was obtained from Iolitec GmbH. PAN-deposition: The PCB board was designed with KiCad-software and manufactured by Aisler B.V. (Germany). PAN-CNT composite films were deposited on the PCB from a $12 \mathrm{wt} \%$ homo-PAN solution in a mixture of DMSO:Acetone $\left(V_{D M S O}: V_{\text {Acetone }}=4: 1\right)$, containing 3 wt $\%$ CNTs (related to the mass of PAN). $2.5 \mathrm{~mL}$ of the PAN-CNT dispersion were deposited in a grid of droplets (microdrop technologies $\mathrm{GmbH}$, Germany) between the collector electrodes over a length of $3 \mathrm{~cm}$. A film was produced on the PCB, by moving a doctor blade (with a distance of $30 \mu \mathrm{m}$ between the blade and the substrate) through the reservoir. The resulting film had a length of $3-6 \mathrm{~cm}$ and a width of $3-2 \mathrm{~cm}$, covering the current-collector and the space in between them. The coated PCB dried for $10 \mathrm{~min}$. at $110^{\circ} \mathrm{C}$ prior to lasercarbonization.

Carbonization: Laser-carbonization was conducted with a high-precision laser engraver setup (Speedy 100, Trotec) equipped with a $60 \mathrm{~W} \mathrm{CO}_{2}$ laser. The laser beam was focused with a 2.5 
inch lens providing a focal depth of $\sim 3 \mathrm{~mm}$ and a focus diameter of $170 \mu \mathrm{m}$. The center wavelength of the laser is $10.6 \pm 0.03 \mu \mathrm{m}$. The laser was operated a pulsed vector mode at 1000 $\mathrm{Hz}$. Therefore, the resulting energy input onto the film (fluence) is given per distance in $\mathrm{J} \cdot \mathrm{m}^{-1}$. The areal energy density results from the effective line width $d(170 \mu \mathrm{m})$ of the carbonized structures obtained in the laser focus. The scanning speed $v^{\prime}$, generically given in $\%$, was converted into $\mathrm{s} \cdot \mathrm{m}^{-1}$. The effective output power $P$ of the laser was measured with a Solo 2 (Gentec Electro-Optics) power meter. The line separation for the patterning of the interdigitated electrodes was $75 \mu \mathrm{m}$.

$$
F=P \cdot v^{\prime} \cdot d^{-1}
$$

The unexposed, non-carbonized PAN-film was removed by peeling off.

Electrochemical measurements: The produced microsupercapacitors were connected to an Iviumsat.h potentiostat (Ivium technologies) via banana clips. All measurements were performed in a glovebox, with a two-electrode setup and $20 \mu \mathrm{L}$ of EMIM TFSI as an electrolyte. The electrolyte covered the whole electrode structure. The cyclic voltammograms were recorded at scanrates from $5-1000 \mathrm{mV} / \mathrm{s}$. The specific capacitance was calculated by $C_{\mathrm{sp}}=$ $\frac{\int_{-1 V}^{1 V} I d V}{2 \cdot v \cdot 2 V \cdot A}$, with the scanrate $v$ and the electrode area $A$. Impedance spectra were recorded from $5 \mathrm{MHz}$ to $0.01 \mathrm{~Hz}$ at a voltage of $0.1 \mathrm{~V}$.

Instruments: IR-spectra were recorded on a PerkinElmer Spectrum two. SEM images were recorded on a Hitachi S-5200.

\section{Supporting Information}

Supporting Information is available from the author.

\section{Acknowledgements}

We thank Carsten Johnigk and Dr. Christian Vedder both at ILT - Fraunhofer Institute for Laser Technology, Aachen, Germany for measuring the laser absorbance of PAN-CNT dispersions. The authors thankfully acknowledge funding from the BMBF SuperCarbon (13XP5036E) and the Cluster of Excellence EXC2154 POLiS.

\section{References}

(1) Huang, P.; Lethien, C.; Pinaud, S.; Brousse, K.; Laloo, R.; Turq, V.; Respaud, M.; Demortière, A.; Daffos, B.; Taberna, P. L.; et al. On-Chip and Freestanding Elastic Carbon Films for Micro-Supercapacitors. Science (80-. ). 2016, 351, 691-695. 
(2) Kyeremateng, N. A.; Brousse, T.; Pech, D. Microsupercapacitors as Miniaturized Energy-Storage Components for on-Chip Electronics. Nat. Nanotechnol. 2017, 12, 7 15.

(3) Bu, F.; Zhou, W.; Xu, Y.; Du, Y.; Guan, C.; Huang, W. Recent Developments of Advanced Micro-Supercapacitors: Design, Fabrication and Applications. npj Flex. Electron. 2020, 4.

(4) Jia, R.; Shen, G.; Qu, F.; Chen, D. Flexible On-Chip Micro-Supercapacitors: Efficient Power Units for Wearable Electronics. Energy Storage Mater. 2020, 27, 169-186.

(5) Lee, Y.; Bandari, V. K.; Li, Z.; Medina-Sánchez, M.; Maitz, M. F.; Karnaushenko, D.; Tsurkan, M. V.; Karnaushenko, D. D.; Schmidt, O. G. Nano-Biosupercapacitors Enable Autarkic Sensor Operation in Blood. Nat. Commun. 2021, 12, 4-13.

(6) Mayorga-Martinez, C. C.; Moo, J. G. S.; Khezri, B.; Song, P.; Fisher, A. C.; Sofer, Z.; Pumera, M. Self-Propelled Supercapacitors for On-Demand Circuit Configuration Based on WS2 Nanoparticles Micromachines. Adv. Funct. Mater. 2016, 26, 66626667.

(7) Ye, J.; Tan, H.; Wu, S.; Ni, K.; Pan, F.; Liu, J.; Tao, Z.; Qu, Y.; Ji, H.; Simon, P.; et al. Direct Laser Writing of Graphene Made from Chemical Vapor Deposition for Flexible, Integratable Micro-Supercapacitors with Ultrahigh Power Output. Adv. Mater. 2018, 30 .

(8) Hourdakis, E.; Nassiopoulou, A. G. Microcapacitors for Energy Storage: General Characteristics and Overview of Recent Progress. Phys. Status Solidi Appl. Mater. Sci. 2020, 217, 1-9.

(9) Han, J.; Lin, Y. C.; Chen, L.; Tsai, Y. C.; Ito, Y.; Guo, X.; Hirata, A.; Fujita, T.; Esashi, M.; Gessner, T.; et al. On-Chip Micro-Pseudocapacitors for Ultrahigh Energy and Power Delivery. Adv. Sci. 2015, 2, 1-7.

(10) Sung, J. H.; Kim, S. J.; Lee, K. H. Fabrication of Microcapacitors Using Conducting Polymer Microelectrodes. J. Power Sources 2003, 124, 343-350.

(11) Eftekhari, A.; Li, L.; Yang, Y. Polyaniline Supercapacitors. J. Power Sources 2017, 347, 86-107.

(12) El-Kady, M. F.; Kaner, R. B. Scalable Fabrication of High-Power Graphene MicroSupercapacitors for Flexible and on-Chip Energy Storage. Nat. Commun. 2013, 4.

(13) Go, D.; Lott, P.; Stollenwerk, J.; Thomas, H.; Moeller, M.; Kuehne, A. J. C. Laser Carbonization of PAN-Nanofiber Mats with Enhanced Surface Area and Porosity. ACS Appl. Mater. Interfaces 2016, 8, 28412-28517. 
(14) Go, D.; Opitz, M.; Lott, P.; Rahimi, K.; Stollenwerk, J.; Thomas, H.; Möller, M.; Roling, B.; Kuehne, A. J. C. Electrochemical Characterization of Laser-Carbonized Polyacrylonitrile Nanofiber Nonwovens. J. Appl. Polym. Sci. 2018, 135, 46398.

(15) Delacroix, S.; Wang, H.; Heil, T.; Strauss, V. Laser-Induced Carbonization of Natural Organic Precursors for Flexible Electronics. Adv. Electron. Mater. 2020, 6, 2000463.

(16) Strauss, V.; Anderson, M.; Turner, C. L.; Kaner, R. B. Fast Response Electrochemical Capacitor Electrodes Created by Laser-Reduction of Carbon Nanodots. Mater. Today Energy 2019, 11, 114-119.

(17) Nataraj, S. K.; Yang, K. S.; Aminabhavi, T. M. Polyacrylonitrile-Based Nanofibers - A State-of-the-Art Review. Prog. Polym. Sci. 2012, 37, 487-513.

(18) Zhang, L.; Aboagye, A.; Kelkar, A.; Lai, C.; Fong, H. A Review: Carbon Nanofibers from Electrospun Polyacrylonitrile and Their Applications. J. Mater. Sci. 2014, 49, 463-480.

(19) Wang, H.; Delacroix, S.; Osswald, O.; Anderson, M.; Heil, T.; Lepre, E.; Lopez-salas, N.; Kaner, R. B.; Smarsly, B. Laser-Carbonization : Peering into the Formation of Micro-Thermally Produced ( N-Doped ) Carbons. Carbon N. Y. 2021, 176, 500-510.

(20) Mei, B. A.; Munteshari, O.; Lau, J.; Dunn, B.; Pilon, L. Physical Interpretations of Nyquist Plots for EDLC Electrodes and Devices. J. Phys. Chem. C 2018, 122, 194206. 